\title{
AUTOCUIDADO, ALFABETIZACIÓN EN SALUD Y CONTROL GLICÉMICO EN PERSONAS CON DIABETES MELLITUS TIPO 2
}

\author{
SELF-CARE, HEALTH LITERACY AND GLYCEMIC CONTROL IN \\ PEOPLE WITH DIABETES MELLITUS TYPE 2
}

\begin{abstract}
AUTOCUIDADO, ALFABETIZAÇÃO EM SAÚDE E CONTROLE GLICÊMICO EM PESSOAS COM DIABETES MELLITUS TIPO 2
\end{abstract}

\author{
Natalia Aguayo-Verdugo* \\ SANDra ValenZuela-Suazo* \\ Luis LUENGO-MACHUCA ${ }^{* * *}$
}

\section{RESUMEN}

Objetivo: Evaluar si existe relación entre autocuidado y alfabetización en salud con control glicémico e identificar sus principales predictores en personas con diagnóstico de Diabetes Mellitus tipo 2, en un Centro de Salud Familiar de Concepción, Chile. Material y Método: Estudio cuantitativo, transversal, descriptivo y correlacional. Participaron 175 personas, quienes dieron respuesta a un instrumento compuesto por: Datos biosociodemográficos, Resumen de Actividades de Autocuidado en Diabetes y Evaluación breve de la alfabetización de salud para adultos hispanohablantes, además glicemia preprandial y HbA1c para verificar control glicémico. Resultados: Los participantes fueron en su mayoría mujeres (70,3\%); edad promedio 65,1 años; años de escolaridad promedio 9,3 años; con pareja el 61,7\%; pensionados el 45,7\%; ingreso económico per cápita $\$ 147.300$ aproximadamente; tiempo transcurrido desde el diagnóstico, 11 años, aproximadamente; utilizando antiglicémicos orales el $63,4 \%$ y no fumadores un $88,6 \%$. Tres áreas de autocuidado clasificaron como inadecuadas: alimentación, actividad física y automonitorización de glicemia; $20 \%$ con inadecuada alfabetización en salud, existiendo diferencia en el nivel educacional, ingreso económico y tipo de terapia farmacológica; $65,8 \%$ con control glicémico inadecuado. Conclusiones: No se obtuvo relación entre autocuidado y alfabetización en salud con control glicémico. Se identificó como principal predictor del control glicémico el tipo de terapia farmacológica. Se evidenció un autocuidado inadecuado y un bajo porcentaje de compensación, lo que lleva a reflexionar sobre el quehacer como equipo de salud en la atención primaria, debiendo movilizar los esfuerzos a favor del modelo integral para mantener una población compensada y libre de complicaciones.

Palabras clave: Autocuidado; Alfabetización en Salud; Diabetes Mellitus Tipo 2; Control Glicémico; Atención Primaria de Salud; Enfermería.

\footnotetext{
*Enfermera. Universidad de Concepción, Concepción, Chile. Email: Nataliaguayo@udec.cl

**Enfermera. Universidad de Concepción, Concepción, Chile. Email: svalenzu@udec.cl. Autor de correspondencia

***Profesor de física y matemática. Universidad de Concepción, Concepción, Chile. Email: lluengom@udec.cl
} 


\section{ABSTRACT}

Objective: To assess whether there is a relationship between self-care, health literacy with glycemic control and identify its main predictors in people diagnosed with Type 2 Diabetes Mellitus at a Family Health Center in Concepción, Chile. Material and Method: Quantitative, cross-sectional, descriptive and correlational study. 175 people participated responding instruments that consisted of bio-sociodemographic questions, Summary of Self-Care Diabetes Activities and Health Literacy Brief Assessment for Spanish-speaking adults, as well as pre-prandial glycemia and HbA1c to verify glycemic control. Results: Participants were mostly women (70.3\%); average age 65.1 years; average years of schooling 9.3 years; with a partner (61.7\%); pensioners (45.7\%); per capita income, approximately $\$ 147,300$; time since diagnose, approximately 11 years; using oral antiglycemic agents (63.4\%); non-smokers (88.6\%). Three areas of self-care were classified as inadequate: nutrition, physical activity and self-monitoring of glycemia; $20 \%$ of participants showed inadequate health literacy, with differences in educational level, income and pharmacological therapy; 65.8\% with inadequate glycemic control. Conclusions: There was no relationship between self-care and health literacy with glycemic control. The type of pharmacological therapy was identified as the main predictor of glycemic control. There is evidence of inadequate self-care and a low compensation level, which leads to reflect on improving work in primary health care and increasing efforts to have a comprehensive model to maintain a compensated population and free of complications.

Key words: Self-care; Health Literacy; Type 2 Diabetes Mellitus; Primary Health Care; Glycemic Control, Nursing.

\section{RESUMO}

Objetivo: Avaliar se existe relação entre autocuidado, alfabetização em saúde com controle glicêmico e identificar seus principais preditores em pessoas diagnosticadas com Diabetes Mellitus Tipo 2 em um Centro de Saúde da Família em Concepción, Chile. Material e Método: Estudo quantitativo, transversal, descritivo e correlacional. Participaram 175 pessoas que responderam a um instrumento composto por: Perguntas biosociodemográficas, Resumo das atividades de autocuidado em diabetes e Breve avaliação da alfabetização em saúde para adultos de língua espanhola, além de glicemia pre-prandial e HbA1c para verificar o controle glicêmico. Resultados: Os participantes foram majoritariamente mulheres (70,3\%); idade média 65,1 anos; média de anos de escolaridade 9,3 anos; com parceiro o (61,7\%); pensionistas o (45,7\%); renda econômica per capita \$ 147.300 aproximadamente; tempo decorrido desde o diagnóstico, aproximadamente 11 anos; uso de agentes antiglicêmicos orais o $(63,4 \%)$; não fumantes o $(88,6 \%)$. Três áreas de autocuidado classificadas como inadequadas: alimentação, atividade física e automonitoramento da glicemia; $20 \%$ com alfabetização em saúde inadequada, havendo diferença no nível educacional, renda econômica e tipo de terapia medicamentosa; 65,8\% com controle glicêmico inadequado. Conclusôes: Não houve relação entre autocuidado e alfabetização em saúde com controle glicêmico. O tipo de terapia farmacológica foi identificado como o principal preditor do controle glicêmico. Foi evidenciado autocuidado inadequado e baixo percentual de compensação, o que leva a refletir sobre a tarefa como equipe de saúde na atenção básica, e deve mobilizar esforços em favor do modelo integral para manter uma população compensada livre de complicações.

Palavras-chave: Autocuidado; Alfabetização em Saúde; Diabetes Mellitus Tipo 2; Controle glicêmico; Atenção Primária à Saúde; Enfermagem.

Fecha recepción: 06/08/2019

Fecha aceptación: 12/09/2019 


\section{INTRODUCCIÓN}

Según estimaciones, 422 millones de adultos en el mundo tenían Diabetes Mellitus (DM) en 2014, frente a los 108 millones de $1980^{(1)}$. En Chile, los resultados de la Encuesta Nacional de Salud (ENS) 2016-2017 mostraron una prevalencia para DM de $12,3 \%{ }^{(2)}$. Esta enfermedad es una condición crónica de tratamiento complejo, que demanda adherencia de la persona a comportamientos de autocuidado que engloben alimentación saludable, actividad física y uso correcto de la medicación, entre otros ${ }^{(3)}$.

Orem define Autocuidado como "la conducta aprendida por el individuo dirigida hacia sí mismo y el entorno para regular los factores que afectan su desarrollo en beneficio de la vida, salud y bienestar" ${ }^{(4)}$. Destaca, a su vez, que las personas tienen habilidades para satisfacer sus necesidades y sino buscan ayuda en familiares o profesionales ${ }^{(5)}$. Vinculado a la educación para el autocuidado se encuentra el concepto de Alfabetización en Salud (AS), definido, según la Organización Mundial de la Salud (OMS), como "las habilidades sociales y cognitivas que determinan el nivel de motivación y la capacidad de una persona para acceder, entender y utilizar la información de forma que le permita promover y mantener una buena salud"(6). Esta habilidad generalmente permanece estable durante la vida y puede mejorar con educación.

Tanto el Autocuidado como la Alfabetización en salud son fenómenos especialmente críticos en la Diabetes Mellitus, pues el control glicémico ideal, según la Asociación Americana de Diabetes (ADA) significa, para los adultos con DM, cumplir con una $\mathrm{HbA1c}<7 \%$, mantener una glucosa plasmática preprandial $80-130 \mathrm{mg} / \mathrm{dl}$ y una glucosa plasmática posprandial $<180 \mathrm{mg} / \mathrm{dl}^{(7)}$.

Existe un objetivo acordado a nivel mundial para detener el aumento de la DM y obesidad para el año 2025 y debido a esto se creó el Programa de Diabetes de la OMS, cuya misión es prevenir esta enfermedad, minimizar las complicaciones y maximizar la calidad de vida ${ }^{(8)}$. En Chile, los objetivos sanitarios para la década 2011-2020 proponen incrementar la proporción de personas con diabetes controlada y desarrollar estrategias para mejorar la pesquisa y control ${ }^{(9)}$. Además, la DM se encuentra incluida dentro de las Garantías Explícitas en Salud, garantizando su diagnóstico y tratamiento.
Investigaciones internacionales apuntan a resolver el problema de compensación en personas con DM, primeramente, tratando de definir los factores asociados y después creando intervenciones. Osborn et al. ${ }^{(10)}$ mencionan que la AS tiene un efecto indirecto sobre el autocuidado y el control glicémico; Bains y Egede ${ }^{(11)}$ encontraron que la AS está asociada con el conocimiento de la DM y Ariza et al. ${ }^{(12)}$ evidenciaron que la inadecuada AS se asocia con el mal control de la glicemia. En la población estudiada por Van der et al. ${ }^{(13)}$ la baja AS se asoció significativamente con menos conocimiento de la DM, mayor nivel de HbAlc y menos actividad física. Lael-Monfared et al. ${ }^{(14)}$ evidenciaron que los participantes con una puntuación de AS más alta habían practicado más comportamientos de autocuidado.

Si bien se ha levantado información a nivel mundial sobre diversos factores que afectan la compensación en las personas con DM, no se encontraron estudios a nivel nacional que hubieran indagado sobre esta problemática y que incluyeran el estudio de variables como Autocuidado y AS, las cuales son necesarias para mejorar la compensación de estas personas, con la consecuente disminución de la morbimortalidad y todo lo que esto implica para la salud pública. El conocimiento del estado actual de una población objetivo es el punto de inicio que podrá guiar investigaciones posteriores en el tema, por lo que se planteó evaluar si existe relación entre Autocuidado y Alfabetización en salud con el control glicémico e identificar sus principales predictores en personas con diagnóstico de Diabetes Mellitus tipo 2 (DMT2) en un Centro de Salud Familiar (CESFAM) de Concepción, Chile.

\section{MATERIAL Y MÉTODO}

Tipo de Estudio y Unidad de análisis: Estudio cuantitativo, transversal, descriptivo y correlacional $^{(15)}$ cuya unidad de análisis fueron personas mayores de 18 años, beneficiarias del sistema público de salud, usuarias del Programa de Salud Cardiovascular con diagnóstico de DMT2 del CESFAM Dr. Víctor Manuel Fernández de la Comuna de Concepción, Chile. Se eligió este centro de referencia por su tamaño y población adscrita. 
Marco muestral: El cálculo del tamaño de la muestra se basó en el trabajo de Peduzzi et al. ${ }^{(16)}$, quienes plantearon una fórmula para el número de casos en regresión logística: [ $\mathrm{N}=10 * \mathrm{k} / \mathrm{p}$ ], siendo "p" la menor de las proporciones de casos negativos o positivos en la población y " $\mathrm{k}$ " el número de variables independientes. Se consideraron los datos estadísticos del CESFAM seleccionado del año 2016, donde el porcentaje de pacientes con DM con un inadecuado control glucémico fue de 57,1\%* y existen 10 variables a estudiar, por lo tanto $[\mathrm{N}=$ $10 * 10 / 0,571]=175,1$. Criterios de inclusión: Exámenes sanguíneos vigentes: glicemia preprandial y HbA1c, disponibles en el sistema electrónico con una antigüedad máxima de 6 meses, ya que según el Ministerio de Salud de Chile (MINSAL) las personas con diabetes deben mantener controles de salud dos veces al año en la atención primaria ${ }^{(17)}$. Criterios de exclusión: Presentar alguna discapacidad mental o física que le impidieran seguir un tratamiento prescrito y/o responder al instrumento, personas institucionalizadas, analfabetas, con dependencia severa, alteración de la salud mental, demencia, enfermedades terminales o complicaciones graves derivadas de la DM. Se utilizó un muestreo no probabilístico por conveniencia ${ }^{(15)}$, pues el establecimiento posee un sistema de tarjetones con información confidencial, por lo que, sin consentimiento, no se tuvo acceso a los datos de interés y obligó a recolectar la información mediante consulta directa.

Recolección de datos: El instrumento recolector de datos se conformó de tres partes: 1) Cuestionario de elaboración propia para datos biosociodemográficos (edad, sexo, años de escolaridad, situación de pareja, situación ocupacional actual, ingreso económico y exámenes sanguíneos); 2) Resumen de Actividades de Autocuidado en Diabetes (Summary of Diabetes Self-Care Activities o SDSCA), para medir las prácticas de autocuidado en DM (alimentación, ejercicio, automonitorización de glicemia capilar, autocuidado de los pies, adherencia a medicamentos y tabaquismo $)^{(18)}$, un puntaje menor a 5 días corresponde a "autocuidado inadecuado" y mayor o igual a 5 días a "autocuidado adecuado"(19). El instrumento original reportó un alfa de Cronbach de $0,68^{(18)}$ y de 0,63 en la versión adaptada para Colombia $^{(20)}$. Esta última fue utilizada, previa evaluación por expertos y prueba piloto, para realizar ajustes a la población chilena, en donde junto a establecer un tiempo de aplicación aproximado de 30 min, se observó que el término "glucometría" fue la palabra más compleja de comprender y por aquellos pacientes que utilizaban sólo dieta y ejercicio para lograr la compensación se tuvo que agregar el ítem "no corresponde" a las preguntas sobre medicación. Con todo, se seleccionó por ser una escala integral que mide 6 áreas de autocuidado, a diferencia de otros instrumentos disponibles. Se tomó resguardo de los posibles sesgos de comprensión mediante la explicación de palabras que pudieran no ser tan conocidas por parte de los encuestadores hacia los participantes; 3) Evaluación breve de la alfabetización de salud para adultos hispano-hablantes (Short Assessment of Health Literacy for Spanishspeaking Adults o SAHLSA-50), para evaluar la capacidad de entender términos médicos comunes. Un puntaje menor o igual a 37 puntos representa "inadecuada alfabetización en salud" y mayor a 37 "adecuada alfabetización en salud”(21). Reportó un alfa de Cronbach de 0,92 en su versión original ${ }^{(21)}$ y en la versión adaptada para Chile tuvo un buen resultado en el coeficiente de K-Richarson $(\mathrm{KR} 20=0,9255)^{(22)}$. Aunque la literatura ${ }^{(23)}$ advierte que pudiera existir relación entre alguna de las variables presentadas que pudiera originar sesgos que se deben considerar al momento de analizar los resultados, por ejemplo, el nivel educacional y la AS, esto no fue observado en la presente investigación.

Para asegurar la calidad de los datos se realizó una capacitación de 1 h de duración a la enfermera y a las estudiantes de 4to año de Enfermería que aplicaron el instrumento, además de supervisiones semanales. Para el reclutamiento se consultó a usuarios que estuvieran a la espera de atención, previa explicación del proceso, si accedían a participar mediante la firma del consentimiento informado. Se comenzó preguntando sobre los datos biosociodemográficos, se aplicó el SDSCA y el SAHLSA-50, finalizando con la revisión de los exámenes sanguíneos. Todos los participantes fueron informados sobre los

*REM-P4. Población en control programa de salud cardiovascular (PSCV). Hoja de estadística año 2016. Facilitado por Enfermera del CESFAM Dr. Víctor Manuel Fernández. 
procedimientos y objetivos del estudio, respetando las normas éticas concordantes con la Declaración de Helsinki $^{(24)}$ y los 8 principios éticos de Emanuel ${ }^{(25)}$, además se obtuvo la aprobación del Comité Ético Científico del Servicio de Salud Concepción, código 18-04-14.

Análisis de datos: Se realizó un análisis uni y bivariado a través de tablas de frecuencias para variables categóricas y medidas de resumen para variables continuas. Con respecto al SDSCA, para fines estadísticos se invirtieron los valores de las preguntas 4 y 5 con el objetivo de obtener un puntaje global por área, obteniendo 5 áreas: 1) Alimentación general y específica; 2) Actividad física; 3) Automonitorización de glicemia capilar; 4) Autocuidado de los pies y 5) Tratamiento farmacológico. El tabaquismo se midió como presencia/ausencia, por lo tanto, no se pudo obtener un puntaje como en las otras áreas, por lo que no se incluyó como un área en el análisis inferencial. Para la comparación de medias se utilizó prueba $\mathrm{T}$ de student y ANOVA, y para comparar porcentajes se utilizó la prueba de Chi-cuadrado. Como método de determinación de asociación entre las variables se utilizó regresión logística múltiple, y como medida de asociación se usó Odd Ratio (OR), acompañado con su intervalo de confianza. En el modelo final se incluyeron las variables que obtuvieron significancia estadística en el análisis bivariado y se incluyeron variables que fueran relevantes según la literatura y experiencia de los investigadores. Para los contrastes se consideró probabilidad de error tipo I de 0,05 , por ser lo utilizado mayormente por las ciencias de la salud ${ }^{(26)}$. Para procesar los datos se utilizó el software SPSS versión Windows 23.0.

\section{RESULTADOS}

La población estudiada se caracteriza en la Tabla 1 . Se observa una muestra compuesta mayoritariamente por: mujeres (70,3\%); edad promedio 65,1 años (DS 11,0; Mín. 29, Máx. 86); escolaridad promedio 9,3 años (DS 4,2; Mín. 0, Máx. 17); 61,7\% con pareja; 45,7\% pensionados; ingreso económico per cápita de \$147.300 aproximadamente (DS 95.300; Mín. 30.000, Máx. 550.000); aproximadamente 10,78 años de tiempo transcurrido desde el diagnóstico (DS 9,1; Mín. 0,08 Máx. 50); 63,4\% utilizando antiglicémicos orales y no fumadores el $88,6 \%$. En lo que respecta a las variables clínicas se encontró, en promedio, una glicemia preprandial de 53,7 mg/dL (DS 61,3; Mín. 46, Máx. 397) y la HbA1c exhibió un promedio de 7,88 \% (DS 2,1; Mín. 5, Máx. 15,4). Se definió control glicémico adecuado a las personas con glicemia preprandial y HbA1c dentro de rango normal, que correspondió al 34,2\%. Respecto de la alfabetización en salud, los participantes mostraron un puntaje promedio de 41,1 (DS 7,1; Mín. 9, Máx. 50), mostrando un $80 \%$ de la muestra una adecuada alfabetización en salud. Respecto del autocuidado, para cada área se calculó el promedio de días donde realizaban la conducta y el mayor promedio (6,7 días; Mín. 0, Máx. 7) lo obtuvo Tratamiento farmacológico. Cabe destacar que hubo 6 personas que no utilizaban fármacos, por lo tanto, el total fue de 169 personas. Por otro lado, el área con menor promedio fue Automonitorización de glicemia capilar (1,2 días; Mín. 0, Máx. 7).

Al realizar la comparación de la adecuada/ inadecuada Alfabetización en Salud por variables biosociodemográficas, se observa una diferencia estadísticamente significativa en nivel educacional, ingreso económico (Tabla 2) y tipo de terapia farmacológica (Tabla 3).

Para el análisis del control glicémico con AS y autocuidado se utilizó regresión logística, no encontrándose asociación estadísticamente significativa (Tabla 4).

Para conocer los predictores del control glicémico se utilizó regresión logística (Tabla 5). Se encontró una asociación estadísticamente significativa únicamente en Tipo de terapia farmacológica con un $\mathrm{R}$ cuadrado de 0,207 , siendo más probable que las personas con "antiglicémicos orales" tengan un adecuado control glicémico en comparación con "ambos". 
Tabla 1. Distribución de los participantes según variables de estudio $(\mathrm{n}=175)$.

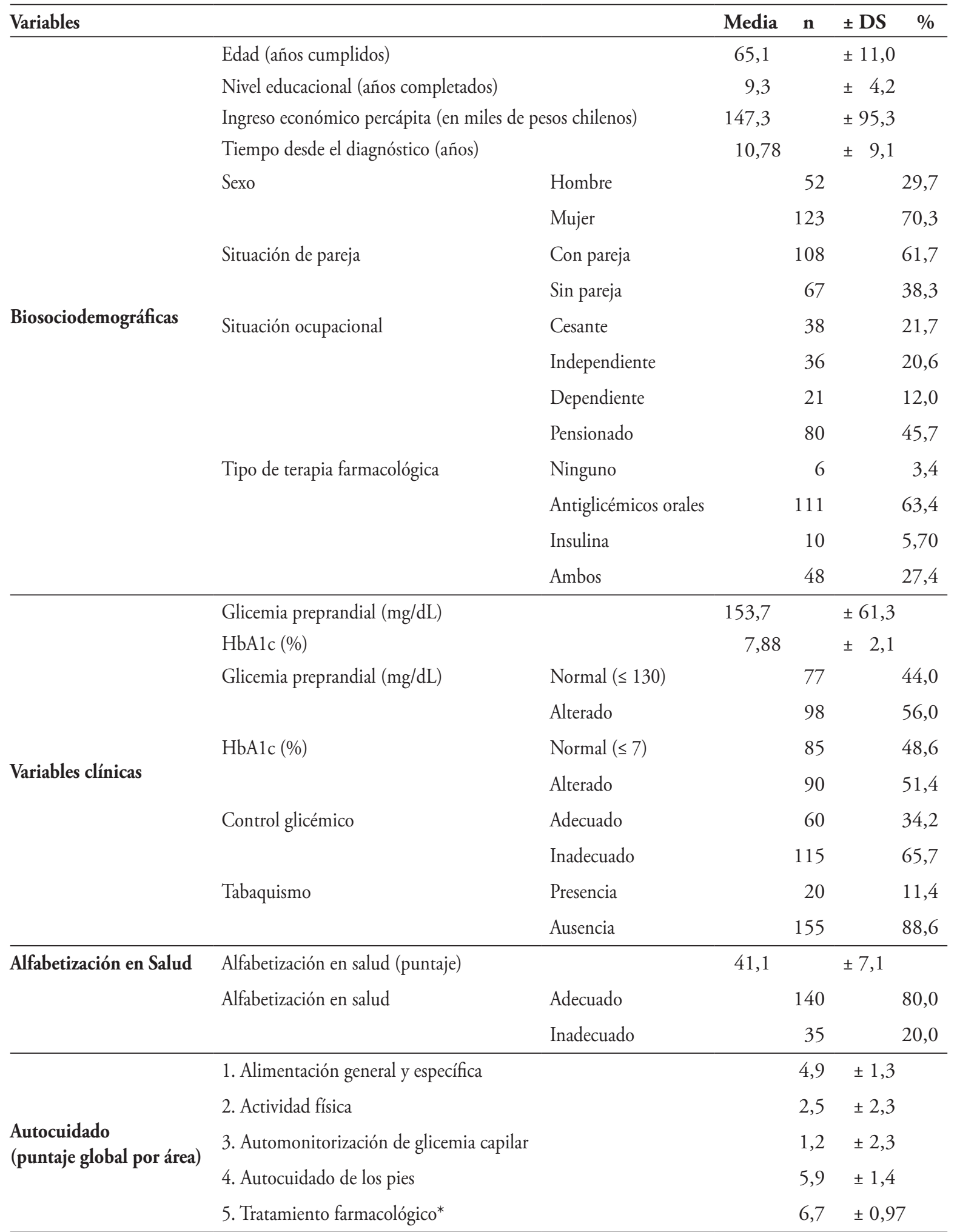

*n=169. DS: desviación estándar; n: número de casos. 
Tabla 2. Distribución de los participantes en función de la Alfabetización en Salud relacionada con variables biosociodemográficas $(n=175)$.

\begin{tabular}{lcrr}
\hline Variables continuas & Adecuada AS & Inadecuada AS & p-valor \\
\cline { 2 - 3 } & Media & Media & \\
\hline Edad (años cumplidos) & 64,5 & 67,5 & 0,145 \\
Nivel educacional (ańos completados) & 9,9 & 6,9 & $<0,001$ \\
Ingreso económico percápita (en miles de pesos chilenos) & 157,2 & 107,7 & 0,006 \\
Tiempo desde el diagnóstico (años) & 11 & 10 & 0,573 \\
\hline
\end{tabular}

Tabla 3. Distribución de los participantes en función de la Alfabetización en Salud relacionada con variables biosociodemográficas $(\mathrm{n}=175)$.

\begin{tabular}{|c|c|c|c|c|}
\hline \multirow{2}{*}{ Variables categóricas } & & \multirow{2}{*}{$\begin{array}{c}\text { Adecuada AS } \\
\text { n (\%) }\end{array}$} & \multirow{2}{*}{$\begin{array}{c}\text { Inadecuada AS } \\
\text { n (\%) }\end{array}$} & \multirow{2}{*}{ p-valor } \\
\hline & & & & \\
\hline \multirow[t]{2}{*}{ Sexo } & Hombre & $38(73,1)$ & $14(26,9)$ & 0,151 \\
\hline & Mujer & $102(82,9)$ & $21(17,1)$ & \\
\hline \multirow[t]{2}{*}{ Situación de pareja } & Con pareja & $86(79,6)$ & $22(20,4)$ & 0,876 \\
\hline & Sin pareja & $54(80,6)$ & $13(19,4)$ & \\
\hline \multirow[t]{4}{*}{ Situación ocupacional } & Cesante & $34(89,5)$ & $4(10,5)$ & 0,372 \\
\hline & Independiente & $27(75,0)$ & $9(25,0)$ & \\
\hline & Dependiente & $17(81,0)$ & $4(19,0)$ & \\
\hline & Pensionado & $62(77,5)$ & $18(22,5)$ & \\
\hline \multirow[t]{4}{*}{ Tipo de terapia farmacológica } & Ninguno & $3(50,0)$ & $3(50,0)$ & 0,023 \\
\hline & Antiglicémicos orales & $92(82,9)$ & $19(17,1)$ & \\
\hline & Insulina & $5(50,0)$ & $5(50,0)$ & \\
\hline & Ambos & $40(83,3)$ & $8(16,7)$ & \\
\hline
\end{tabular}

n: número de casos.

Tabla 4. Relación entre Alfabetización en salud y Autocuidado con control glicémico ( $\mathrm{n}=169)$.

\begin{tabular}{lcccccc}
\hline Areas & B & Error estándar & Wald & gl & p-valor & OR \\
\hline Alfabetización en salud & $-0,025$ & 0,079 & 0,10 & 1 & 0,750 & 0,975 \\
Alimentación & $-0,011$ & 0,355 & 0,00 & 1 & 0,975 & 0,989 \\
Actividad física & 0,113 & 0,202 & 0,31 & 1 & 0,577 & 1,119 \\
Automonitorización de glicemia capilar & $-0,085$ & 0,148 & 0,332 & 1 & 0,564 & 0,918 \\
Autocuidado de los pies & $-0,447$ & 0,287 & 2,431 & 1 & 0,119 & 0,640 \\
Tratamiento farmacológico & $-0,331$ & 0,516 & 0,412 & 1 & 0,521 & 0,718 \\
Tabaquismo & $-0,774$ & 1,467 & 0,278 & 1 & 0,598 & 0,461 \\
Constante & 4,825 & 5,570 & 0,75 & 1 & 0,386 & 124,579 \\
\hline
\end{tabular}

R cuadrado de Nagelkerke: 0,205. 
Tabla 5. Predictores del Control glicémico $(\mathrm{n}=169)$.

\begin{tabular}{|c|c|c|c|c|c|c|c|c|}
\hline \multirow[t]{2}{*}{ Variables } & \multirow[t]{2}{*}{ B } & \multirow[t]{2}{*}{ Error estándar } & \multirow[t]{2}{*}{ Wald } & \multirow[t]{2}{*}{ gl } & \multirow[t]{2}{*}{ p-valor } & \multirow[t]{2}{*}{ OR } & \multicolumn{2}{|c|}{ 95\% C.I. para OR } \\
\hline & & & & & & & Inferior & Superior \\
\hline Sexo & 0,568 & 0,406 & 1,952 & 1 & 0,162 & 1,764 & 0,796 & 3,910 \\
\hline Tto fco (1) & $-1,693$ & 1,134 & 2,231 & 1 & 0,135 & 0,184 & 0,020 & 1,697 \\
\hline Tto fco (2) & $-2,353$ & 1,311 & 3,224 & 1 & 0,073 & 0,095 & 0,007 & 1,240 \\
\hline Tto fco (3) & $-3,299$ & 1,204 & 7,512 & 1 & $0,006^{*}$ & 0,037 & 0,003 & 0,391 \\
\hline Edad & 0,021 & 0,017 & 1,488 & 1 & 0,222 & 1,021 & 0,987 & 1,056 \\
\hline Ingreso económico & 0,003 & 0,002 & 3,648 & 1 & 0,056 & 1,003 & 1,000 & 1,007 \\
\hline Alimentación & $-0,161$ & 0,140 & 1,319 & 1 & 0,251 & 0,851 & 0,646 & 1,121 \\
\hline Actividad física & 0,028 & 0,082 & 0,119 & 1 & 0,730 & 1,029 & 0,876 & 1,207 \\
\hline Constante & $-0,208$ & 1,721 & 0,015 & 1 & 0,904 & 0,812 & & \\
\hline
\end{tabular}

${ }^{*} \mathrm{p}<0,05$. T to fco (1): Tipo de terapia farmacológica "antiglicémicos orales". Tto fco (2): Tipo de terapia farmacológica “insulina”. Tto fco (3): Tipo de terapia farmacológica "ambos".

\section{DISCUSIÓN}

Comparado con otros estudios a nivel nacional, el perfil de la muestra es similar ${ }^{(27,28)}$ y destacó un menor porcentaje de hombres en comparación con las mujeres. Un estudio realizado en Chile plantea que los hombres tienen una menor percepción de riesgo, por lo cual no consultan sino hasta que los problemas de salud se han agudizado ${ }^{(29)}$. En cuanto a la situación ocupacional, predominaron las categorías "pensionado" y "cesante", coincidiendo con un estudio realizado en Santiago de Chile, cuyos investigadores señalan como posible causa la incompatibilidad de los horarios de atención del centro de salud con la jornada laboral ${ }^{(30)}$.

Respectoalautocuidado, las3áreas bajo el puntaje considerado adecuado: Alimentación general y específica, Actividad física y Automonitorización de glicemia capilar, y las 2 áreas con autocuidado adecuado: Autocuidado de los pies y Tratamiento farmacológico, se corresponde con lo encontrado en un estudio mexicano ${ }^{(19)}$ en el que la alimentación y la actividad física fueron áreas de autocuidado con puntajes bajos, a pesar de la importancia que tienen ambas en el control metabólico. Los hidratos de carbono son fundamentales, ya que determinan hasta en un $50 \%$ la variabilidad en la respuesta glicémica $^{(31)}$ y el ejercicio aeróbico y de fuerza mejoran el control glicémico, reducen factores de riesgo cardiovascular y mejoran la condición física, entre otros ${ }^{(32)}$. Sorprende que personas con un diagnóstico de enfermedad crónica no realicen actividades en favor de mantener su salud y prevenir complicaciones, teniendo la creencia de que el tratamiento farmacológico por sí solo es suficiente. Estos resultados se corresponden con la población general chilena que alcanza un $86,7 \%$ de sedentarismo y solo un $15 \%$ de consumo de 5 porciones de frutas y verduras al día ${ }^{(2)}$. Cabe destacar que la Automonitorización de la glicemia capilar no está dentro de los lineamientos de atención primaria en Chile, ya que es un gasto que no se justifica en usuarios compensados ${ }^{(17)}$, lo que estaría explicando el bajo nivel encontrado.

Con respecto a la $\mathrm{AS}$, en este estudio fue adecuada en el $80 \%$, lo cual se contrapone con un estudio argentino, donde se encontró una adecuada AS en un $39,7 \% \%^{(12)}$. Esta discordancia se podría explicar por las diferencias en el nivel educacional, pues en ese estudio más del $50 \%$ de los participantes tenían 7 o menos años de educación formal. Una evidencia de lo anterior es que el instrumento utilizado para medir AS permite captar errores de conocimiento, que en algunos casos son de conceptos fundamentales, por ejemplo, algunas personas eligen "sal" en lugar de "azúcar" para relacionarlo con el concepto "diabetes". Esto motiva a reflexionar sobre la educación que se brinda a la población, pues poseer conocimientos de salud se convierte en un reto primordial para garantizar que las personas tengan un mayor control de su propia salud y con ello un mayor bienestar. En Europa, 
la AS está considerada como una medida clave en la estrategia europea de salud para el 2020, siendo un tema complejo, ya que cada vez más personas acceden a la información disponible en internet ${ }^{(33)}$. Según un estudio realizado el 2014, seis de cada diez europeos consultaban internet para resolver dudas sobre problemas de salud, y nueve de cada diez confiaban en la información que encuentran allíi ${ }^{(34)}$. Esto se constituye en un desafío a abordar y no debe ser visto como una desventaja sino más bien como una necesidad de potenciar las estrategias en e-health, cuidando el nivel de AS digital para garantizar el acceso a información de calidad.

En esta muestra resultó significativo el nivel educacional, observándose que las personas con adecuada AS tenían en promedio tres años más de educación formal en comparación con las personas con inadecuada AS. Se reafirma entonces, en este estudio, que el nivel educativo es uno de los factores más asociados con la alfabetización en salud ${ }^{(35,36)}$. A su vez, existió una diferencia estadísticamente significativa en el ingreso per cápita, donde hubo promedio de $\$ 50.000$ pesos chilenos más en las personas con adecuada AS versus las personas con inadecuada AS. Jiménez y Ortega ${ }^{(37)}$ mencionan que existen diversos estudios que encuentran que en la práctica la autovaloración del nivel de salud, la tasa de mortalidad o el nivel de enfermedades crónicas, entre otros indicadores de salud, tienen una relación inversa con la posición socioeconómica, lo cual se observa en esta muestra, donde a menor ingreso económico fue menor la probabilidad de presentar una adecuada AS.

$\mathrm{Al}$ indagar sobre los predictores del control glicémico se encontró mayor probabilidad que los participantes con "antiglicémicos orales" tengan un adecuado control glicémico en comparación con "ambos". Según los lineamientos del MINSAL, las personas con DM inestable deben utilizar metformina e insulina ${ }^{(38)}$, por lo tanto, existe más probabilidad que la persona que está utilizando ambos tratamientos esté descompensada.

Se destaca el rol de las variables biosociodemográficas en la explicación del fenómeno, lo cual demuestra la importancia de los determinantes sociales al momento de brindar atención como equipo de salud, coincidiendo con otro estudio chileno, en donde la edad, sexo, educación e ingresos económicos se asociaron con un estilo de vida promotor de salud ${ }^{(39)}$.
Orem menciona que cuando la persona no puede llevar a cabo conductas de autocuidado para mantenerse compensado es cuando interviene el profesional de Enfermería, para educar y enseñar a tomar decisiones responsables ${ }^{(40)}$. En este sentido, los controles cardiovasculares deben representar una oportunidad para la educación y promoción del autocuidado, generando metas en conjunto (plan consensuado) $)^{(41)}$. Aun así, en la práctica se observa una fragmentación del cuidado en salud, es decir, una desconexión entre el cuidado realizado por los diferentes profesionales tanto del área médica, como de enfermería y nutrición. Si bien desde el 2017 existe la Orientación Técnica Programa de Salud Cardiovascular ${ }^{(42)}$, no todos los profesionales están al tanto de los nuevos lineamientos, por lo que es fundamental realizar capacitaciones en el tema y reuniones de sector para unificar criterios y fomentar la comunicación entre el equipo, especialmente en casos complejos.

Aunque se trabajó con el concepto "Autocuidado" por ser el constructo con más investigaciones asociadas a DM, se recomienda, en futuras investigaciones, trabajar con el concepto "Automanejo", ya que, si bien son términos usados de manera similar, se han realizado esfuerzos para diferenciarlos ${ }^{(43)}$. Automanejo se define como "las intervenciones, entrenamiento y habilidades por las cuales los pacientes con una cronicidad, invalidez o enfermedad pueden cuidar de ellos mismos eficazmente y pueden aprender cómo hacerlo" ${ }^{(44)}$. Autocuidado es utilizado para las personas sin patologías crónicas ${ }^{(44)}$.

Finalmente, la principal limitación del presente estudio fue la utilización de un instrumento no validado para la población chilena (SDSCA), sin embargo, se tomaron los resguardos mencionados en el apartado "material y método". Se sugiere la validación de este instrumento para futuras investigaciones.

\section{CONCLUSIONES}

Con respecto al objetivo de la investigación no se encontró relación entre autocuidado y AS con control glicémico, sin embargo, se identificó como principal predictor del control glicémico el tipo de terapia farmacológica. Lo más probable es que las personas que utilizan de forma aislada 
antiglicémicos orales tengan un adecuado control glicémico en comparación a las personas que utilizan la combinación de antiglicémicos orales con insulina.

El presente estudio contribuye con evidencia respecto a la situación actual de la población con DMT2 a nivel local, evidenciando un autocuidado inadecuado, especialmente en

\section{REFERENCIAS}

1. Organización Mundial de la Salud. Informe mundial sobre la diabetes: resumen de orientación [Internet]. Ginebra: Organización Mundial de la Salud; 2016 Abr [citado 10 Abr 2019]. 4 p. Disponible en: https://apps.who.int/iris/handle/10665/204877

2. Ministerio de Salud. Encuesta nacional de salud 2016-2017. Primeros resultados. [Internet]. Santiago (CL): Departamento de Epidemiología, División de Planificación Sanitaria, Subsecretaría de Salud Pública; 2017 nov [citado 15 mar 2019]. Disponible en: http://web.minsal.cl/wp-content/ uploads/2017/11/ENS-2016-17_PRIMEROSRESULTADOS.pdf

3. Rezende D, Silva A, Silva G. Adesão das pessoas com diabetes mellitus ao autocuidado com os pés. Rev bras enferm. 2015; 68(1): 111-6.

4. Rivera L. Autocuidado y capacidad de agencia de autocuidado. Av enferm. 2006 jul-dic [citado 15 mar 2019]; 24(2): 91-98. Disponible en: https:// revistas.unal.edu.co/index.php/avenferm/article/ view/35969

5. Vega O, González D. Teoría del déficit del autocuidado: interpretación desde los elementos conceptuales. Rev Ciencia y cuidado. 2007; 4(1): 28-35.

6. Organización Mundial de la Salud. Promoción de la salud, Glosario [Internet]. Ginebra: Unidad de Promoción de la Salud y Educación para la Salud de la División de comunicación, Educación y Promoción de la Salud; 1998. [citado 15 mar 2019] Disponible en: http://whqlibdoc.who.int/hq/1998/ WHO_HPR_HEP_98.1_spa.pdf

7. American Diabetes Association. Control de la glucosa [Internet]. 2015 jun [citado 16 mar 2019]. Disponible en: http://www.diabetes.org/es/vivircon-diabetes/tratamiento-y-cuidado/el-control-dela-glucosa-en-la-sangre/control-de-la-glucosa.html

8. Organización Mundial de la Salud. Programa de la OMS [Internet]. 2016 [citado 16 mar 2019]. Disponible en: https://www.who.int/diabetes/es/

9. Ministerio de Salud. Estrategia nacional de salud las áreas de Alimentación, Actividad física y Automonitorización de la glicemia capilar, además de un bajo porcentaje de compensación, lo que lleva a reflexionar sobre el quehacer del equipo de salud en la atención primaria, especialmente del área de Enfermería, encargada de movilizar los esfuerzos en favor del modelo integral para mantener una población compensada y libre de complicaciones.

para el cumplimiento de los Objetivos Sanitarios de la Década 2011-2020 [Internet] Chile [citado $16 \mathrm{mar}$ 2019]. Disponible en: https://www.minsal.cl/portal/ url/item/c4034eddbc96ca6de0400101640159b8.pdf

10. Osborn C, Bains S, Egede L. Health Literacy, Diabetes Self-Care, and Glycemic Control in Adults with Type 2 Diabetes. Diabetes Technol Ther. 2010 nov; 12(11): 913-9.

11. Bains S, Egede L. Associations Between Health Literacy, Diabetes Knowledge, Self-Care Behaviors, and Glycemic Control in a Low Income Population with Type 2 Diabetes. Diabetes Technol Ther. 2011 mar; 13(3): 335-41.

12. Ariza A, Lanteri E, Cícero C, Pérez A, Puchulu F, Mejía R. Alfabetización en salud y control de la diabetes en pacientes de un hospital universitario de Argentina. Medicina (B. Aires). 2017 Jun; 77 (3): 167-172.

13. Van der Heide I, Uiters E, Rademakers J, Struijs JN, Schuit AJ, Baan CA. Associations among health literacy, diabetes knowledge, and self-management behavior in adults with diabetes: results of a dutch cross-sectional study. J Health Commun. 2014; 19(Supl 2): 115-31.

14. Lael-Monfared E, Tehrani H, Esmati Z, Ferns G, Tatari M, Jafari A. Health literacy, knowledge and self-care behaviors to take care of diabetic foot in low-income individuals: Application of extended parallel process model. Diabetes \& Metabolic Syndrome: Clinical Research \& Reviews; 13 (2019): 1535-1541.

15. Hernández R, Fernández C, Baptista P, Méndez S. Metodología de la investigación. $6^{a}$ ed. México D.F. 2014. 126-194 p.

16. Peduzzi P, Concato J, Kemper E, Holford T, Feinstein A. A simulation study of the number of events per variable in logistic regression analysis. J Clin Epidemiol. 1996; 49(12): 1373-1379.

17. Ministerio de Salud. Guía clínica Diabetes Mellitus tipo 2 [Internet]. Santiago (CL):2010. [citado 20 mar 2019]. Disponible en: http://web.minsal.cl/portal/ url/item/72213ed52c3e23d1e04001011f011398. pdf 
18. Toobert D, Hampson S, Glasgow R. The summary of Diabetes Self-Care Activities Measure. Diabetes Care. 2000; 23(7): 943-950.

19. Flores-Pérez M, Garza-Elizondo M, HernándezCortés P. Autocuidado en el adulto mayor con diabetes tipo 2. Monterrey, México. Rev enferm Herediana. 2015; 8(2): 70-74.

20. Uribe D, Peńa R. Validación del Cuestionario de Actividades de Autocuidado en Diabetes (SDSCA) para Colombia [Tesis]. 2015. [citado 17 mar 2019]. 30p. Disponible en: https://repositorio.uniandes. edu.co/bitstream/handle/1992/12960/u713476. pdf?sequence $=1$ \&isAllowed $=y$

21. Lee S, Bender D, Ruiz R. Development of an Easy to-Use Spanish Health Literacy Test. Health Serv Res. 2006 Aug [citado 18 mar 2019]; 41(4 Pt 1): 1392-1412. Disponoble en: doi: 10.1111/j.14756773.2006.00532.x

22. Monsalves M, Mañalich J, Fuentes E. Validación del test Short Assessment of Health Literacy for Spanishspeaking Adults en Chile, para medir alfabetización en salud. Rev méd Chile [Internet]. 2016 may [citado 18 mar 2019]; 144 (5): 604-610. Disponible en: https://scielo.conicyt.cl/scielo.php?script=sci_ arttext\&pid=S0034-98872016000500008

23. Stormacq C, Van den Broucke S, Wosinski J. Does health literacy mediate the relationship between socioeconomic status and health disparities? Integrative review. Health Promot Int. 2019; 34 (5): 1-17.

24. Barrios I, Anido V, Morera M. Declaración de Helsinki: cambiosy exégesis. Rev CubanaSalud Pública [Internet]. 2016 mar [citado 18 mar 2019]; 42 (1): 132-142. Disponibleen: http://scielo.sld.cu/scielo.php?script=sci_ arttext\&pid=S0864-34662016000100014

25. Emanuel EJ, Wendler D, Killen J, Grady C. What Makes Clinical Research in Developing Countries Ethical? The Benchmarks of Ethical Research. J Infect Dis. 2004 mar; 189(5): 930-7.

26. Manterola C, Pineda V. El valor de "p" y la "significación estadística": Aspectos generales y su valor en la práctica clínica. Rev Chil Cir. 2008 feb; 60 (1): 86-89.

27. Urzúa A, Chirino A, Valladares G. Autorreporte de la calidad de vida relacionada con la salud en diabetes mellitus tipo 2. Rev méd Chile [Internet]. $2011 \mathrm{mar}$ [citado 18 mar 2019]; 139 (3): 313-320. Disponible en: https://scielo.conicyt.cl/scielo.php?script=sci_ arttext\&pid=S0034-98872011000300005

28. Lange I, Campos S, Urrutia M, Bustamante C, Alcayaga C, Tellez A. et al. Efecto de un modelo de apoyo telefónico en el auto-manejo y control metabólico de la Diabetes tipo 2, en un Centro de Atención Primaria, Santiago, Chile. Rev méd Chile [Internet]. 2010 jun [citado 18 mar 2019]; 138 (6): 729-737. Disponible en: https://scielo.conicyt.cl/scielo.php?script=sci_ arttext\&pid=S0034-98872010000600010

29. Ministerio de Salud. Situación actual de la salud del hombre en Chile [Internet]. Subsecretaría de salud pública, División de Prevención y Control de Enfermedades, Departamento de Ciclo Vital: 2011 [citado 18 mar 2019] Disponible en: https://www.minsal.cl/portal/url/item/ b7e8f68be82d7f2fe040010165013351.pdf

30. Mora J. Creencias y motivaciones de un grupo de diabéticos del CESFAM Cardenal Raúl Silva Henríquez, para abandonar un programa de actividad física [Tesis]. Santiago: Universidad de Chile; 2014 [citado 12 dic 2019]. 101 p. Disponible en: http://bibliodigital.saludpublica.uchile.cl:8080/ dspace/bitstream/handle/123456789/374/Tesis_ Julia\%20Mora.pdf?sequence $=1$ \&isAllowed $=y$

31. Durán S, Carrasco E, Araya M. Alimentación y diabetes. Nutr Hosp. 2012 ago; 27 (4): 1031-1036.

32. Márquez JJ, Suárez R, Márquez J. El ejercicio en el tratamiento de la diabetes mellitus tipo 2. RAEM [Internet]. 2012 [citado 20 mar 2019]; 49 (4): 203212. Disponible en: http://academia.utp.edu.co/ alejandrogomezrodas/files/2016/09/Ejercicio-en-elTratamiento-de-la-Diabetes.pdf

33. Juvinyà-Canal D, Bertran-Noguer C, SuñerSoler R. Alfabetización para la salud, más que información. Gac Sanit [Internet]. 2018 feb [citado 10 abr 2019]; 32 (1): 8-10. Disponible en: http://scielo.isciii.es/scielo.php?script=sci_ arttext\&pid=S0213-91112018000100008

34. European Union. European citizens' digital health literacy 2014 [Internet]. 2014 [citado 10 abr 2019]. Disponible en: http://ec.europa.eu/ commfrontoffice/publicopinion/flash/fl_404_ en.pdf.

35. Ruiz-Cabello AL. La alfabetización en salud de la población española: Variables relacionadas según los resultados del proyecto europeo de alfabetización en salud [tesis doctoral]. Murcia: Universidad de Murcia. Facultad de Medicina; 2015 [citado 11 abr 2019]. Disponible en: https://www.tesisenred.net/ handle/10803/309753\#page $=1$

36. Ariza A, Lanteri M, Cicero C, Pérez A, Puchulu F, Mejía R. Alfabetización en salud y control de la diabetes en pacientes de un hospital universitario de argentina. MEDICINA (Buenos Aires). [Internet]. 2017 [citado 11 abr 2019]; 77: 167-172. Disponible en: https://www.medicinabuenosaires.com/volumen77-ano-2017/volumen-77-ano-2017-no-3-indice/ alfabetizacion-en-salud-y-control-de-la-diabetes-enpacientes-de-un-hospital-universitario-de-argentina/

37. Jiménez D, Ortega M. Salud y posición socioeconómica: evidencia empírica reciente en el panorama internacional y en España. ReE. 
[Internet]. 2014 jul [citado 12 abr 2019]; 0 (1). Disponible en: https://revistaselectronicas.ujaen.es/ index.php/REE/article/view/1369

38. Ministerio de Salud: Guía de Práctica Clínica Tratamiento Farmacológico de la Diabetes Mellitus tipo 2 [Internet]. 2016-2017 [citado 10 abr 2019]. Disponible en: http://www. soched.cl/guias/2017.08.10_DIABETES\%20 MELLITUS\%20TIPO\%202\%20FINALISIMA. pdf

39. Vidal D, Chamblas I, Zavala G, Müller R, Rodríguez M, Chávez A. Determinantes sociales en salud y estilos de vida en población adulta de Concepción, Chile. Cienc enferm [Internet]. 2014 abr [citado 10 abr 2019]; 20 (1): 61-74. Disponible en: https://scielo.conicyt.cl/scielo.php?script=sci_ arttext\&pid=S0717-95532014000100006

40. Gallegos E, Bañuelos P. Autocuidado y control en adultos mayores con diabetes. Desarrollo Científ Enferm. 2011 may: 9(4): 101.

41. Ministerio de Salud. Visita Domiciliaria Integral Orientaciones Técnicas en el Marco del Modelo de Atención Integral de Salud Familiar y Comunitaria
[Internet]. Subsecretaría de Redes Asistenciales, División de Atención Primaria [citado 10 abr 2019]. Disponible en: https://diprece.minsal.cl/ wp-content/uploads/2018/06/2018.04.17_OTVISITA-DOMICILIARIA-INTEGRAL.pdf

42. Ministerio de salud: Orientación técnica Programa de Salud Cardiovascular [Internet] 2017 [citado $10 \mathrm{abr}$ 2019] Disponible en: http://www.capacitacionesonline. com/blog/wp-content/uploads/2017/09/Programa-desalud-cardiovascular.-MINSAL-Chile-2017.pdf

43. Lange I, Bustamante C, Alcayaga C, Escobar MC, Herrera L, Ferrer L, et al. Del autocuidado al automanejo: la escuela de Enfermería UC en la vanguardia del cuidado crónico. Horiz Enferm [Internet]. 2015 [citado 10 abr 2019]; 26 (2): $37-$ 46. Disponible en: http://horizonteenfermeria. uc.cl/images/pdf/26-2/2_del_autocuidado_al_ automanejo_reflexion.pdf

44. Bonal R, Cascaret X. ¿Automanejo, autocuidado o autocontrol en enfermedades crónicas? Acercamiento a su análisis e interpretación. MEDISAN. 2009; 13(1): 1-11. 